\title{
SUPERCONDUCTIVE TEST CAVITY FOR THE ESS
}

\author{
W. Bräutigam, O. Felden, M. Glende, R. Maier, H. Meier, A. Schnase, G. Schug, H. Singer, \\ R. Stassen*, Forschungszentrum Jülich, Germany
}

\begin{abstract}
Measurements at the prototype cavity were performed at the FZJ Jülich in order to support the design work of the SC accelerator part for the European Spallation Source (ESS) [1]. A shielded test facility has been constructed providing $25 \mathrm{~kW}$ peak RF power operating at $500 \mathrm{MHz}$ [2]. The test cavity has been fabricated at ACCEL Instruments and was installed into the shielded area. This 5-cell cavity has been designed to a $\beta$ value of 0.75 at an accelerating field of $\mathrm{E}_{\text {acc }}>5 \mathrm{MV} / \mathrm{m}$ with $\mathrm{Q}_{0}=2 \times 10^{9}$, operating at $4.2 \mathrm{~K}$. We have developed a cavity control circuit based on a fast analogue phase-lock loop allowing automated measurements. The first quasi static measurements using a $500 \mathrm{~W}$ broadband amplifier almost verified the design values of $\mathrm{Q}_{0}$ and $\mathrm{E}_{\text {acc }}$. The piezoelectric tuning system was used to record the spectrum of mechanical resonances of the cavity. A comparison of several computer simulations with measured results is presented. Furthermore, we have taken dynamical data of the Lorentz force detuning during pulsed-mode operation at a repetition rate of $50 \mathrm{~Hz}$. First results achieved with an active compensation of the Lorentz force detuning using the fast piezo frequency control system are also presented.
\end{abstract}

\section{OPERATIONAL CHARACTERISTICS}

The first RF measurements were done during the acceptance tests of the cavity. We took the $Q_{0}-E_{\text {acc }}$ curve (Fig. 1) at nearly critical coupling in order to measure $Q_{0}$ as accurate as possible.

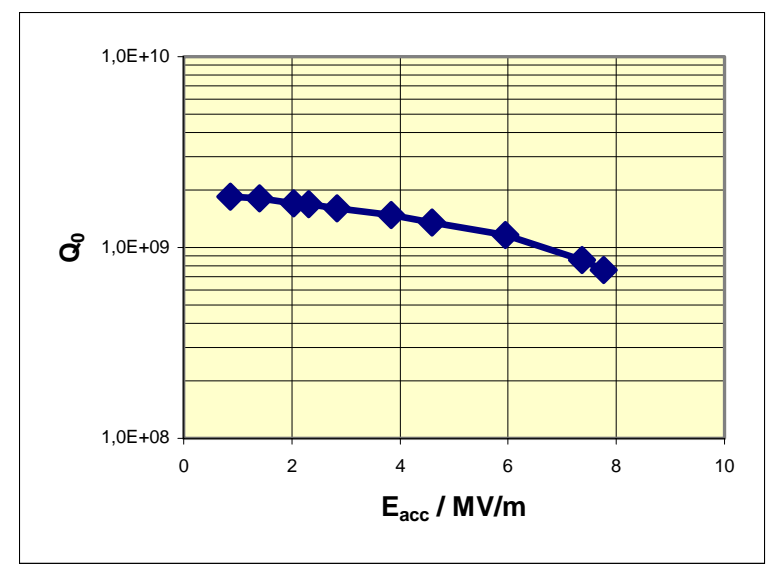

Fig. 1: $\mathrm{Q}_{0}$ measured vs $\mathrm{E}_{\text {acc }}$ of the test cavity.

These results agree well with those measured at CERN in a vertical cryostate and at a limited RF power of $200 \mathrm{~W}$.

*e-mail: r.stassen@fz-juelich.de
Using the 500W amplifier we can achieve accelerating fields up to nearly $8 \mathrm{MV} / \mathrm{m}$ without detecting a quench, but the measured $\mathrm{Q}_{0}$ degradation at increasing $\mathrm{RF}$ field strength and the monitored $\mathrm{x}$-ray levels point to active field emitters inside the cavity. At higher field levels we also saw moving light spots on the vacuum window (glass) which seem to be generated by strong electron loading. Even the mirror installed outside the cavity to allow an indirect view with a camera was tarnished by a brown film. The estimated dose of the mirror is about 30Gy during one minute.

We can switch from the $500 \mathrm{~W}$ amplifier to a SIEMENS $25 \mathrm{~kW}$ transmitter on loan from ACCEL. We checked that transmitter and incorporated it into a high power system with a circulator and a high power switch. That allows tests of the SIEMENS transmitter and simultaneously use the 500W amplifier for cavity tests or vice versa [3].

The input coupler of the cavity is adjustable from nearly critical coupling to a loaded Q of the order of 1E6. We can reach a filling time of about $2 \mathrm{~ms}$ with this coupling and $30 \mathrm{~kW}$ RF power. After filling the cavity and maintaining the flattop by switching back to $7 \mathrm{~kW}$ to simulate the beam, the RF power is switched off $1 \mathrm{~ms}$ later (Fig. 2).

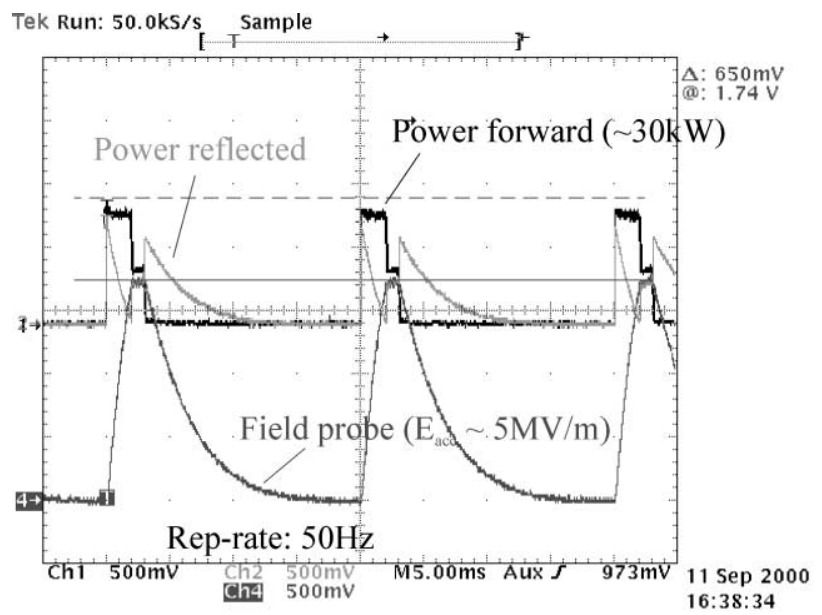

Fig. 2: Pulsed mode operation characteristics.

Even at a repetition rate of $50 \mathrm{~Hz}$, we reached a field-level of about $5 \mathrm{MV} / \mathrm{m}$ in this mode as guaranteed by ACCEL applying a forward power of about $30 \mathrm{~kW}$. We can only operate without the Phase-locked-loop if the microphonic noise is minimized by switching off the cryopump and if the system is appropriately detuned. We have conditioned the coupler and the cavity at this pulse scheme during several hours of operation with the closed Phase-lockedloop. The x-ray radiation detected outside the shielded 
area was commensurably reduced. Nearly all field emitters inside the cavity could be eliminated in this way. Meanwhile we have reached a field level of $11 \mathrm{MV} / \mathrm{m}$ at pulse lengths of about $100 \mathrm{~ms}$ and a repetition rate of $1 \mathrm{~Hz}$.

\section{MECHANICAL RESONANCES}

Higher repetition rates such as $50 \mathrm{~Hz}$ for the ESS design [1] and $60 \mathrm{~Hz}$ at SNS [4] or $75 \mathrm{~Hz}$ for the CERN Neutrino Factory design [5] will require a close look into the mechanical resonances of the cavities. Such mechanical resonances can influence the phase behaviour of the cavity during a pulse, which can hardly be compensated by a good control system, even if enough additional power is available. Microphonic effects are also very dangerous and have to be examined.

We used the three piezo elements provided for the fine frequency adjustment to detect the mechanical resonances. A static electrical tension of $500 \mathrm{~V}$ at the piezos caused a tune shift of $1.2 \mathrm{kHz}$. The first measurements were done with a sinusoidal excitation of the piezo elements. The piezo system is fast and the time response in our system is limited by the used power supplies. We stimulated the piezos up to $200 \mathrm{~Hz}$. Higher frequencies are possible but there is no experience about stress limits of the piezos operating at these frequencies, and their limits by power dissipation. We measured the mixer output signal of the closed frequency control [9] in response to the stimulation and found a dominating resonance at about $120 \mathrm{~Hz}$.

This strong resonance can also be stimulated if we excite at $1 / 2$ or $1 / 3$ of this frequency. Such phenomena subharmonic excitation of mechanical resonances - can be a great problem for future SC proton accelerators. The scanning of all resonances in the frequency domain would be too time consuming. Therefore we applied a step function in the time domain and calculated the resonances using Fast Fourier Transformation (FFT, Fig. 3).

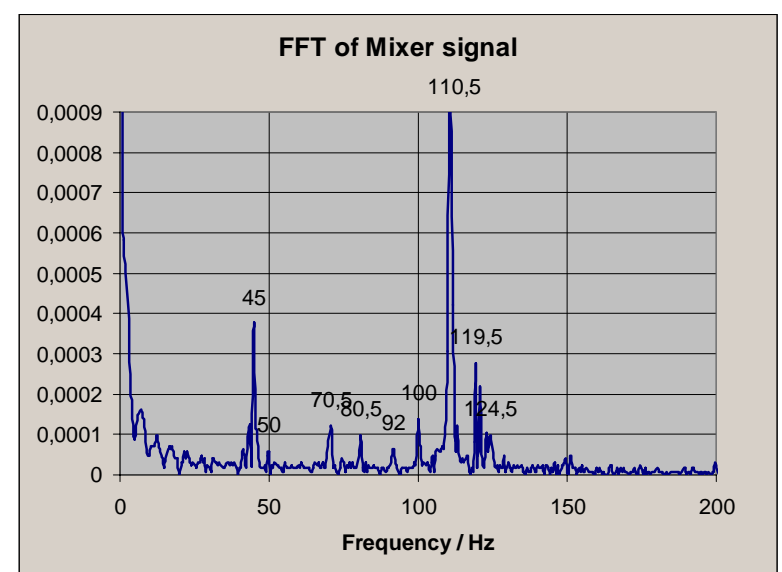

Fig. 3: FFT of the response to a 50V step function at the piezos.

In addition to the mechanical resonances we found the $50 \mathrm{~Hz}$ and $100 \mathrm{~Hz}$ line of the ripple of the AC power line. We were able to detect mechanical resonances even when applying only a low (50V) step function at the piezos.
These measurements have been done stimulating all three piezos in parallel mode. Thus, mostly longitudinal resonances were excited. Even the detection system - the mixer output signal - is very sensitive to longitudinal deformations. Additional measurements are needed to separate longitudinal from transverse modes. This could be done by excitation with a separate system mounted mechanically to the support of the cavity. The three piezos can then be used as a detection system. By means of their different mounting positions, it will be possible to decide if a transverse or longitudinal mode is excited.

Different calculations of the mechanical resonances have been carried out. The first calculation by ACCEL showed the lowest resonance far above $50 \mathrm{~Hz}$ [6]. Further calculations including more details of the cavity in the model seem to be much closer to the measured data [7]. A precise evaluation can only be made after additional measurements, including the possibility to decide the mode of the resonance. Future measurements are planned using the piezo elements as a diagnostic tool to record directly the vibration of the cavity in the $\mu \mathrm{m}$ range. The first measurements have proved the perturbation of the cavity and we have seen a mechanical resonance during filling of the cavity with liquid helium at about 1 to $2 \mathrm{~Hz}$. This resonance can also be seen in the frequency-control circuit and is still not yet understood.

\section{MICROPHONICS}

The cavity RF resonance is sensitive to vibrations of sub- $\mu \mathrm{m}$ amplitude because of the high Q. These microphonic effects cause low-frequency noise in the accelerating fields. Therefore, careful damping of cavity support and other ancillary units is necessary to suppress all vibrations of cavity. High microphonic noise is produced by the 'Gifford McMahon' cryo-generator which is connected to the cavity test cryostat to reduce helium losses [10]. We can see a phase oscillation corresponding to the frequency of the pump (about $1 \mathrm{~Hz}$ ). Even the effects of a turbo-pump can be seen, which demonstrates the sensitivity of the system. The microphonics cannot be reduced by feed-forward because there is no correlation to the pulse repetition rate. Hence vibration-sensitive pick-ups will be installed to build an active damping system in the future. The microphonics caused by the cryopump lead to a strong phase-shaking of more than $\pm 20^{\circ}$. These phase perturbations can not be tolerated in accelerator operation and must be mechanically compensated or regulated by a fast control system.

\section{LORENTZ FORCE DETUNING}

The Lorentz force detuning (LFD) in a short-pulse mode operation is normally described by the constant $\mathrm{K}$ and the mechanical time constant $\tau_{\mathrm{LFD}}$.

The frequency change due to the LFD was measured using the mixer-output signal of the regulation system. We obtained an LFD constant of about $\mathrm{K}=4 \mathrm{~Hz} /(\mathrm{MV} / \mathrm{m})^{2}$. Even at the relatively low design field value $(5 \mathrm{MV} / \mathrm{m})$ of our test cavity, the frequency shift of about $100 \mathrm{~Hz}$ is not 
negligible. The time constant $\tau_{\mathrm{LFD}}$ of the LFD was measured during pulsed-mode operation with a pulselength of about $10 \mathrm{~ms}$. We estimated a time constant of about $5 \mathrm{~ms}$. This high time constant allows a pulse length of $1 \mathrm{~ms}$ like ESS without regulating circuit. Before the LFD takes place, the pulse is descending. For beam acceleration, the phase change could be corrected at lower power levels.

Fig. 4 represents the cavity behaviour of the new pulse scheme for the ESS combining a short pulse $(1 \mathrm{~ms})$ at $50 \mathrm{~Hz}$ and an additional long pulse $(3 \mathrm{~ms})$ each $60 \mathrm{~ms}$.

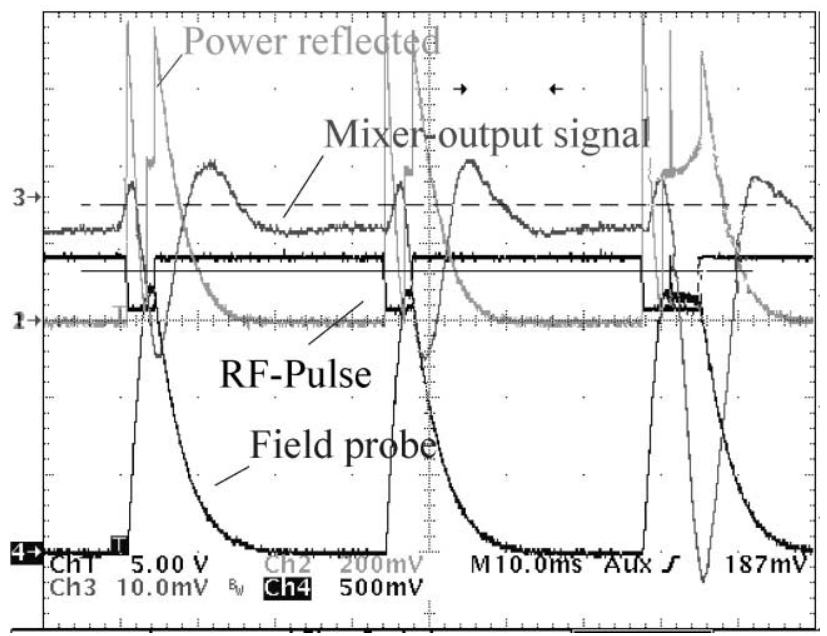

Fig. 4: Phase behaviour (mixer output signal) of the test cavity during an additional long pulse at a accelerating field of $5 \mathrm{MV} / \mathrm{m}$

The LFD leads to a so large phase shift at the long pulse that the flattop field level is descending at the end of the pulse.

\section{Active compensation of Lorenz force detuning}

Stiffening the cavities can reduce Lorentz force detuning but this method will increase the force that is necessary to tune the cavity. One way to solve this problem can be the right choice of the location of the stiffening rings [8]. Another possibility is to detune the cavity and to control only the phase change with a fast control system. In this case large additional klystron power is needed. We tried to compensate the LFD with a feed-forward signal because it is a predictable effect. We used the piezo elements of the fine-tuning system because they act fast enough and can be adjusted precisely. The first approximation to compensate the Lorentz force detuning is shown in Fig. 5. Without feed-forward, we could see the change of the mixer output signal. Using a feedforward signal in the form of one period of a sine wave, the phase stays constant during flattop even at a pulse rate of $50 \mathrm{~Hz}$ as specified in the ESS proposal.

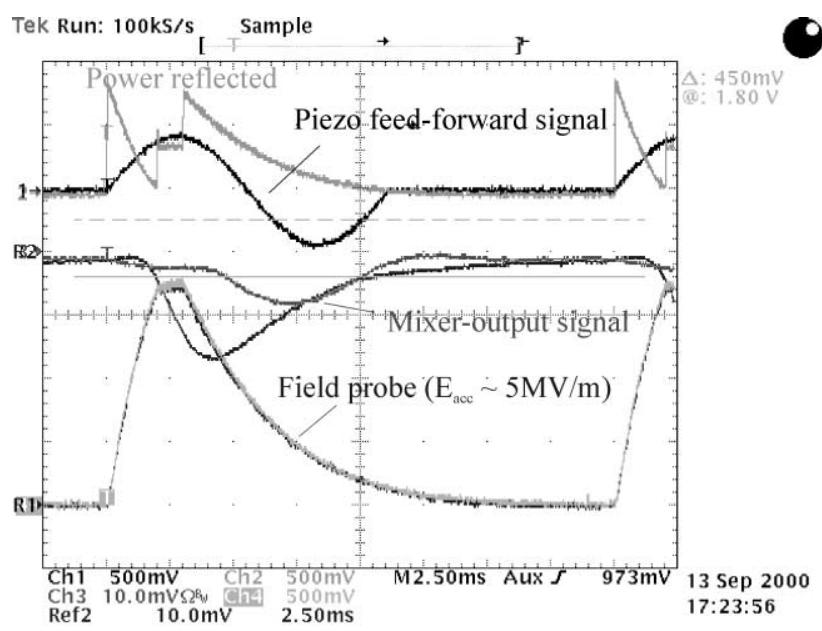

Fig. 5: Compensation of Lorentz force detuning with piezo elements.

\section{OUTLOOK}

The piezos used for the fine frequency tuning of the SC cavity act fast enough to allow a compensation of the Lorentz force detuning with a feed-forward signal. We will perform systematically measurements of the cavity response to different mechanical stimulation. After finding a mechanical-electrical simulation model of the cavity, we will construct and implement an analogue or digital [11] feedback circuit for compensating all possible perturbations.

\section{REFERENCES}

[1] ESS A next Generation Neutron Source for Europe, Volume III, ESS-96-93-M

[2] G.Schug et al, Radiation Protection for ESS Prototype-Cavity Experiments, IKP Ann. Rep. 1998, Jül-3640

[3] A. Schnase, Pulsed power amplifier for ESS test of a $500 \mathrm{MHz}$ superconducting cavity, IKP Ann Rep. 2000, Jül-3852

[4] J.R. Alonso, The Spallation Neutron Source Projekt, PAC99, New York

[5] H. Haseroth, CERN Ideas and Plans for a Neutrino Factory, NuFact'00 Workshop, Monterey

[6] W. Diete et al, A Superconducting Accelerating Test Module for the European Spallation Neutron Source, PAC99, New York

[7] J.F. Stelzer, Analysis of the dynamic behaviour of the ESS superconducting accelerator modules, to be published as ESS Report

[8] C. Pagani, RFS Activities at INFN Milano-LASA, $7^{\text {th }}$ workshop of superconductivity, Saclay

[9] W. Bräutigam et al, ESS Test Cavity: First RF Measurements, IKP Ann Rep. 2000, JÜL-3852

[10]H.Vogel et al, A Superconducting Accelerating Test Module for the European Spallation Neutron Source, PAC99, New York

[11] S. Simrock, Design of the digital RF control system for the TESLA TEST FACILITY, EAPC96, Barcelona 